\title{
(K)eine neue Verfassung für das geeinte Deutschland?
}

\section{Eine $\mathbb{Z}$ wischenbilant nach Abschluß der Beratungen der Gemeinsamen Verfassungskommission}

\section{Stand der Arbeiten an der Verfassungsreform}

Die Gemeinsame Verfassungskommission (GVK) von Bundestag und Bundesrat hat nach fast zweijährigen Beratungen am 28. Oktober 1993 ihren Abschlußbericht (GVK 1993a)' einstimmig beschlossen und damit ihre Arbeit beendet. Die GVK hat in 26 Sitzungen und neun öffentlichen Anhörungen nahezu die Hälfte der Artikel des Grundgesetzes darauf überprüft, ob Änderungen und Ergänzungen notwendig sind. Einer quantitativ so intensiven Durchleuchtung ist das Grundgesetz seit der Enquête-Kommission Verfassungsreform (1972; 1976; dazu Wahl 1978; Grawert 1979) Mitte der 70 er Jahre nicht mehr unterzogen worden. Die von der Kommission mit der vereinbarten Zwei-Drittel-Mehrheit angenommenen Empfehlungen schlagen zu 23 Artikeln Änderungen oder Ergänzungen des Grundgesetzes vor. Die Empfehlungen zu Fragen, die die europäische Einigung an das Grundgesetz stellt und die die verfassungsrechtlichen Konsequenzen aus dem Vertrag von Maastricht ziehen, betreffen acht Artikel und sind bereits Gesetz (Fischer 1993; zur GVK-Empfehlung Scholz 1992). In seiner Sitzung vom 17. Dezember 1993 hat der Bundesrat einstimmig einen Gesetzentwurf zur Umsetzung der weiteren von der Gemeinsamen Verfassungskommission von Bundestag und Bundesrat beschlossenen Empfehlungen verabschiedet. Ein gleichlautender interfraktioneller Antrag, getragen von der SPD und den Koalititionsfraktionen, ist im Januar dem Bundestag vorgelegt worden. Bereits im Dezember 1993 hat die SPD-Bundestagsfraktion

1 Dazu auch die Beiträge im Schwerpunktheft Aus Politik und Zeitgeschichte, Nr. B 5252/93 (24.12.1993) sowie die Thernenausgabe von Das Parlament Nr. 2/1994 vom 14. Januar 1994. Über Verlauf und Ergebnisse der Beratungen s.a. die Berichte von Rubel 1992; 1993a; 1993b. Eine Bilanz aus politikwissenschaftlicher Sicht zieht Benz (1993). An die Beratungen in der GVK knüpfen an ein interfraktioneller Gesetzentwurf von CDU/ CSU, SPD und FDP (BT-Drs. 12/6633 = BR-Drs. 886/93, Beschluß), der die Empfehlungen der GVK umsetzt, sowie weitergehende Änderungsvorsch!äge von SPD (BTDrs. 12/6323) Bündnis 90/Grüne (12/6686 und 6716) und PDS/LL (BT-Drs. 12/6570), ferner ein von über 350 Mitgliedern des Bundestages aus allen Fraktionen und Gruppen unterzeichneter Antrag zu »Mitmenschlichkeit und Gemeinsinn« (BT-Drs. 12/6708). 
ihre Vorstellungen für eine Modernisierung des Grundgesetzes als Gesetzentwurf eingebracht, die in der GVK nicht die erforderliche Mehrheit gefunden hatten. Mitte Januar haben Bündnis 90/Die Grünen und PDS/LL nachgezogen. Diese Gesetzentwürfe sind am 4. Februar 1994 nach der 1. Lesung in die Fachausschüsse verwiesen worden. Eine Beschlußfassung noch vor der parlamentarischen Sommerpause wird angestrebt.

Mit der Aufnahme der parlamentarischen Beratungen im förmlichen $\mathrm{Ge}$ setzgebungsverfahren beginnt eine neue Phase der Verfassungsreformdebatte. Eine Zwischenbilanz aus diesem Anlaß muß den Blick auch zuriick auf die Anstöße dieser Diskussion wenden. Auftrag und Ausgangspunkt für die Beratungen der GVK war formell Artikel 5 des Einigungsvertrages mit der Empfehlung der Regierungen der beiden Vertragsparteien - der Bundesrepublik Deutschland und der DDR - an die gesetzgebenden Körperschaften des vereinten Deutschlands, sich innerhalb einer bestimmten Frist mit den in Zusammenhang mit der deutschen Einigung aufgeworfenen Fragen zur Änderung oder Ergänzung des Grundgesetzes zu befassen. Hervorgehobene Themen sind das Verhältnis zwischen Bund und Ländern, die Möglichkeiten einer Neugliederung für den Raum Berlin/ Brandenburg, Überlegungen zur Aufnahme von Staatszielbestimmungen in das Grundgesetz sowie die Frage einer »Anwendung « des Artikels 146 GG und in deren Rahmen eine Volksabstimmung.

\section{Gründe für eine Verfassungsdebatte}

Sachlich reichen die Anstöße über den unmittelbar durch die deutsche Einigung bedingten Verfassungsänderungsbedarf weit hinaus (s.a. Schneider 1991; Kuratorium 1990; FES 1991; Heuer/Riege 1992; Kammradt 1992; Häberle 1992a; Preuß 1991). Schon deswegen geht der Vorwurf des verhinderten Bundespräsidentschaftskandidaten Heitmann - unter dem Titel Die mißbrauchte Einheit zusammengefaßt (1994) - ins Leere, die deutsche Einheit sei nur Mittel zum Zwecke für eine aus anderen Motiven gespeiste Verfassungsdiskussion gewesen. Mit der politischen Entscheidung, die staatliche Einigung Deutschlands nicht über den Weg einer Neukonstituierung nach Art. $146 \mathrm{GG}$, sondern durch den Beitritt nach Art. $23 \mathrm{GG}$ a.F. zu vollziehen (Isensee 1990; Wiederin 1992; Guggenberger/Stein 1991), ist das Grundgesetz zur in ganz Deutschland geltenden Verfassung geworden. Einigungsinduzierter Änderungsbedarf ergibt sich gleichwohl aus der Frage, ob das auf die Probleme der westlichen Bundesländer zugeschnittene und fortgeschriebene Grundgesetz den veränderten Anforderungen an eine gesamtdeutsche Verfassung - sowohl was ihre zukunftsgestaltende Kraft als auch was ihre Integrationswirkung betrifft - noch genügt. Durch die 
staatliche Einigung Deutschlands sind weiterhin die bisherigen Souveränitätsbeschränkungen der Bundesrepublik Deutschland weggefallen: Die Erlangung der vollen Souveränität erfordert eine neue Verortung der Rolle Deutschlands in der Welt.

Die staatliche Einigung Deutschlands hat weiterhin Menschen mit unterschiedlichen politischen Erfahrungen, aber auch Vorstellungen über eine gerechte Verfassungsordnung zusammengeführt. Ihre spezifischen Erfahrungen fordern einigungsinduzierte Änderungen (Kloepfer 1994) auch dann, wenn Themen und Lösungsangebote an im Westen entwickelte Modelle anknüpfen. Durch ihre Wahlentscheidung am 18. März 1990 hat die Bevölkerung der DDR diese Erfahrungen und Vorstellungen nicht über Bord geworfen und für eine bedingungslose Übernahme eines unveränderten Grundgesetzes votiert: Diese Sicht (Isensee 1993; Kirchhof 1994) unterstellt zu Unrecht, daß dieselben Bürgerinnen und Bürger, die gerade eine alte politische Ordnung friedlich zum Einsturz gebracht haben, auf die aktive Gestaltung der zukünftigen Ordnung des geeinten Deutschlands hätten verzichten wollen. Die staatliche Einigung ist auch bei de jure unbestreitbarer Fortgeltung des Grundgesetzes materiell keine bloße Erweiterung des Verfassungsgebietes. All dies gebot eine umfassende, thematisch nicht beschränkte Verfassungsdiskussion. Zu fragen war nicht - negativ -, welche Prinzipien des Grundgesetzes sich nicht bewährt haben und einigungsbedingt zu ändern wären, sondern - positiv - danach, inwieweit sich Prinzipien des Grundgesetzes verdienen, in die Verfassung eines geeinten Deutschlands aufgenommen zu werden (Seifert 1993).

Neben die deutsche Einigung trat der aus dem Prozeß der europäischen Einigung resultierende Änderungsbedarf. Das Zusammenwachsen Europas, die Entwicklung hin zum europäischen Bundesstaat, hat durch den Vertrag von Maastricht eine neue Qualität erhalten, die Verfassungsbedarf nicht nur wegen des vereinbarten Kommunalwahlrechts für EG-Bürgerinnen und Bürger und der Entscheidung für das europäische Wirtschaftssystem ausgelöst hat: Zu klären war, inwieweit die Europäische Gemeinschaft bzw. die Europäische Union noch vom Modell der »zwischenstaatlichen Einrichtung « des Art. 24 GG a.F. gedeckt waren, welche Anforderungen an die demokratische, rechtsstaatliche, soziale und förderative Struktur des vereinten Europas zu stellen waren, in das sich die Bundesrepublik Deutschland als gleichberechrigtes Glied einfügen kann, und welche Auswirkungen der Integrationsprozeß auf die bundesstaatliche Ordnung und die Rolle der Länder im politischen Prozeß hat (Hofmann 1993). Diesen Problemen hat sich die Gemeinsame Verfassungskommission wegen des Zeitplanes für Maastricht vorab und - hierin stimmen alle Beteiligten überein - in ihrer innovativsten Phase angenommen (GVK 1992). Die Überlagerung partei- 
politischer Fronten durch die spezifischen Interessen der Länder sowie der Einigungsdruck, der wegen der für den Vertrag von Maastricht erforderlichen Zwei-Drittel-Mehrheit erzeugt wurde, haben hier mit der Festlegung eines Staatszieles zur europäischen Integration, einer Struktursicherungsklausel sowie stärkeren Mitwirkungsrechten des Bundesrates zu bereits in das Grundgesetz eingefügten Regelungen geführt, die einen Bestand der Bundesländer in einem förderal aufgebauten Europa sichern helfen sollen. ${ }^{2}$ Die sozialen und demokratischen Defizite Europas, insbesondere auch das demokratische Defizit in den Entscheidungsstrukturen der EG, konnten durch eine Grundgesetzänderung nicht bewältigt werden, zumal sich eine gesamteuropäische politische Kultur (Demokratievorstellungen, Parteien, gesamteuopäische öffentliche Meinung etc.) als Unterbau des demokratischen Prozesses noch nicht herausgebildet hat (Seifert 1992a).

Ein von den Prozessen der deutschen und europäischen Einigung unabhängiger Modernisierungsbedarf ergab sich weiterhin auch aus den veränderten gesellschaftlichen Bedingungen, auf die das Grundgesetz bezogen ist. Im institutionellen Gefüge war das Bund-Länder-Verhältnis zu rejustieren, in dem sich die Gewichte schleichend immer mehr zugunsten des Bundes verschoben hatten (Landtag NRW 1990; Eicher 1988). Dies hätte Reföderalisierungsmaßnahmen auch unabhängig davon erfordert, daß die Kompetenzverlagerung auf die EG einerseits, die höhere Zahl der Länder und das Hinzutreten fünf finanzschwacher, im Aufbau befindlicher und von Bundeshilfen abhängiger Länder das Problem zusätzlich verschärft haben. Wachsende Parteien- und Politikverdrossenheit legten aber auch Maßnahmen, wie sie bereits von der Enquête-Kommission 1976 empfohlen worden sind, im Bereich des Parlamentsrechts nahe, ${ }^{3}$ die zu mehr Offenheit und Transparenz sowie Einwirkungsmöglichkeiten der Bürgerinnen und Bürger führen. Im Interesse einer Stärkung demokratischer Vielfalt stand die 1976 von der Enquête-Kommission noch abgelehnte Ergänzung der bislang auf Bundesebene strikt repräsentativen parlamentarischen Demokratie um Elemente direkter Demokratie im Raum. Die von einer breiten Volksbewegung getragene friedliche Revolution in der DDR bestätigt lediglich, daß

2 Dies war ein wesentliches Anliegen auch der zur Vorbereitung der Verfassungsdebatte vom Bundesrat eingesetzten $\gg$ Kommission Verfassungsreform"; zu deren Ergebnissen $\mathrm{s}$. den Bericht »Stärkung des Föderalismus in Deutschland und Europa sowie weitere Vorschläge zur Änderung des Grundgesetzes«. BR-Drucks. $360 / 92$ vom 14.5.1992; zu deren Ergeonissen s. Asmussen/Eggeling 1993.

3 Einen Innovationsschub, der Vorbild auch für zahlreiche Regelungen in den ostdeutschen Landesverfassungen war, hat hier die auf dem Schlußbericht der vom Landtag SchleswigHolstein eingesetzten Enquete-Kommission Verfassungs- und Parlamentsreform (BadenBaden 1989) gründende neue Verfassung des Landes Schleswig-Holstein bewirkt. 
das in den Strukturen des Grundgesetzes institutionalierte Mißtrauen in die politische Reife des eigenen Volkes nicht gerechtfertigt ist.

Modernisierungsbedarf wegen gesellschaftlicher Veränderungen ergab sich allerdings auch in den Bereichen der Staatsziele und Grundrechte. Dem Staat sind - als planendem und lenkenden Sozialstaat - mehr und mehr Aufgaben zugewachsen (Grimm 1990a). Die gesellschaftliche Ordnung ist wieder selbst zum Gegenstand staatlicher Veränderung und aktiver Gestaltung geworden (Grimm 1990b). Damit verbunden ist eine Überwindung des klassisch liberalen Verfassungsverständnisses, das von einer klaren Trennung von Staat und Gesellschaft ausgeht und die Grundrechte als reine Abwehrrechte gegen den Staat begreift. Konzeptionell ist darauf zu reagieren, daß die rechtliche Verbürgung grundrechtlicher Freiheit allein die tatsächlichen Voraussetzungen des Freiheitsgebrauches nicht sichert und die Menschen in ihrer Existenz auf staatliche Vorsorge und Leistungen angewiesen sind (Böckenförde 1991). Zugleich prägt die Erfahrung entsprechende Verfassungserwartungen, daß die Befriedigung der elementaren Lebensbedürfnisse - Eigensicherung des Lebensunterhaltes durch Erwerbsarbeit, angemessene Versorgung mit menschenwürdigem Wohnraum, soziale Sicherheit bei Krankheit, Unfall, Alter und anderen Wechselfällen des Lebens sowie der Zugang zur Bildung und zum kulturellen Leben - nicht automatisch eintretende Ergebnisse der Wirtschaftsordnung sind, sondern voraussetzungsvolles Ergebnis staatlicher Politik. Gerade in Zeiten sozialer Gefährdung wird es dann aber umso wichtiger, in der Verfassung nicht nur im Detail zu regeln, wie der Staat organisiert ist, sondern durch Ausformung der sozialen Staatsziele deutlich zu machen, daß das Bestreben um soziale Sicherheit und Gerechtigkeit genuine Aufgabe des Staates selbst dann ist, wenn er sie wegen der strukturellen Grenzen staatlicher Tätigkeit letztlich nicht zu garantieren vermag.

Das geschärfte Bewußtsein für die negativen Folgen ökologisch unverträglichen Wachstums und die Gefährdung der natürlichen Lebensgrundlagen verlangt ferner, sich auch in der Verfassung der Erhaltung der Natur anzunehmen, um den einfachgesetzlichen Spielraum für allein von der Verfassung nicht zu garantierende Umweltpolitik zu öffnen. Hier wird seit über zehn Jahren um eine Verfassungsergänzung gerungen (Müller-Bromley 1990; Bock 1990; Robert 1993). Der technische Fortschritt und die Entwicklung hin zu einer Informationsgesellschaft haben neue Freiheitsgefährdungen bewirkt, denen durch grundrechtlichen Datenschutz zu begegnen ist. ${ }^{4}$ Die Pluralisierung der Formen des gesellschaftlichen Zusammenlebens

4 Die Konferenz der Datenschutzbeauftragten hatte in einer Entschließung vom 28.4.1992 u.a. die Aufnahme eines Datenschutzgrundrechts, die Verankerung einer unabhängigen Datenschutzkontrolle und von Informationszugangsrechten empfohlen (Abdruck u.a. im 
erfordern, den privilegierenden und ausgrenzenden Verfassungsschutz von Ehe und Familie auf andere Formen des Zusammenlebens zu erstrecken; aufzugreifen sind die Schwierigkeiten, Familie und Beruf miteinander zu vereinbaren (Berghahn 1993; Herdegen 1993). Ausdifferenzierung der Gesellschaft und Pluralisierung lassen Minderheitenschutzprobleme und Diskriminierungen deutlicher hervortreten. Dies gilt etwa für die Benachteiligung der wachsenden Zahl behinderter Menschen (Herdegen 1992; A. Jürgens 1993), aber auch für die Diskriminierung wegen der sexuellen Identität (Bruns 1993). Grenzüberschreitende Mobilität und Integrationsprozesse haben Fragen der Achtung der ethnischen, kulturellen oder sprachlichen Identität auf Dauer im Lande lebender Ausländerinnen und Ausländer wichtiger werden lassen (Franke/Hofmann 1993; Hofmann 1992; Frowein u.a. 1993); die moderne Massen- und Mediengesellschaft macht aber auch die Minderheiten und Volksgruppen deutscher Staatsangehörigkeit zunehmend von staatlichem Schutz und staatlicher Förderung abhängig, wollen sie ihre kulturelle Identität wahren. Zusätzliche Anforderungen an die Verfassung ergeben sich - last not least - aus der Erfahrung der Frauen, daß die rechtliche Gleichberechtigung von Männern und Frauen faktische, soziale Benachteiligungen nicht hindert und einer faktischen Gleichstellung der Frauen aktiver Fördermaßnahmen bedarf (Limbach/Eckertz-Höfer 1993; Gerhard 1991; Bumke 1993; Deter 1993).

Die Reihe der Beispiele für einen Modernisierungsbedarf des Grundgesetzes ließe sich fortsetzen. Dabei geht es - entgegen Mutmaßungen aus konservativer Sicht (Heitmann 1993) - weder um den Versuch, parteipolitische Forderungen zum Verfassungsgesetz zu erheben, noch - so eine Kritik von links (Günther 1991) - darum, politische Ohnmacht oder mangelnden Gestaltungswillen durch verfassungsrechtliche Vorgaben zu kompensieren. Berührt sind jeweils Themen von grundlegender Bedeutung für das gesellschaftliche Zusammenleben und die Strukturierung und Orientierung staatlicher Macht. Sie bilden zugleich den inhaltlichen Maßstab, an dem der Output der Verfassungskommission zu messen ist.

\section{Grundgesetzånderungen jenseits der GVK: Die Musilk der Machét} spielt anBerhall

Eine Zwischenbilanz zur Verfassungsreformdiskussion kann sich nicht auf Arbeit und Ergebnisse der Gemeinsamen Verfassungskommission beschränken. Das Grundgesetz ist bereits aus Anlaß der deutschen Einigung

21. Tätigkeitsbericht des Hessischen Datenschutzbeauftragen, vorgelegt zum 31.12.1992, 146i.). 
selbst geändert worden (Herdegen 1991): Neugefaßt wurde die Präambel, der Beitrittsartikel 23 GG a. F. wurde gestrichen, die Stimmenverhältnisse im Bundesrat wurden - wie einige sagen, zu Lasten der ostdeutschen, aber auch der kleineren westdeutschen Länder - geändert, übergangsbedingte Abweichungen vom Grundgesetz zugelassen und durch Änderung des Art. 146 unter Beibehaltung der Ablösungsmöglichkeit selbst klargestellt, daß die Bundesrepublik Deutschland als Staat territorial saturiert ist und keinerlei Gebietsansprüche gegen Nachbarn stellt.

Artikel 5 des Einigungsvertrages verbietet den im verfassungsrechtlichen Schrifttum (Kirchhof 1994, 24) gezogenen Schluß, »(j)ede Einzelbestimmung des Grundgesetzes « sei »demnach vor der Einigung geprüft und im wesentlichen für geeignet befunden worden, als Verfassungsgrundlage des vereinten Deutschland - als Einheitsverfassung - zu dienen«. Es stellt die historischen Prozesse auf den Kopf, in Art. 5 EV lediglich das Werk der West-SPD oder ihres seinerzeitigen Kanzlerkandidaten (so Bartlsperger 1990; Blumenwitz 1991; Roellecke 1991) bzw. der westdeutschen Bundesländer zu sehen (so Heitmann 1994).

Nach Aufnahme der Arbeit der GVK ist das Grundgesetz immerhin noch viermal geändert worden. Die Entscheidung über die weitgehende Beschränkung des verfassungshistorisch einmaligen individuellen Grundrechts auf Asyl (ZDFW 1993; Kokott 1993; Hailbronner 1993) ist dabei in der GVK behandelt (GVK 1993b), politisch aber außerhalb der GVK entschieden und nicht in ein Gesamtkonzept der Restrukturierung der Verfassungsordnung eingebettet worden. Die SPD hat sich hier - wie beim Lauschangriff - von der Union in die Defensive treiben lassen: Während die Union in der GVK unter Hinweis auf die bewährten Strukturen des Grundgesetzes jede Modernisierung abgelehnt hat, hat sie durch geschickt inszenierte Kampagnen recht erfolgreich verfassungsgesetzliche Hindernisse konservativer, obrigkeitsstaatlicher Politik abzubauen versucht (Seifert 1993, 92). Von konservativer Seite (Isensee 1993, 2586) wird dagegen die Asylrechtsänderung als »Verfassungsimpuls von unten « bewertet, in dem der »Unterschied deutlich wird zwischen einer vom Volk her kommenden verfassungspolitischen Bewegung, in der die vitale Macht des pouvoir constituant unterschwellig wirksam ist, und einer von der politischen Klasse inszenierten, einer künstlich herbeigeredeten Verfassungsdebatte $\ll$. An der GVK weitgehend vorbeigelaufen sind weiterhin die zur Umsetzung der »Reformen« bei Bahn und Post erforderlichen Verfassungsänderungen; die Privatisierungsentscheidungen haben Signalwirkung unabhängig davon, ob damit langfristig der Staat an Steuerungsfähigkeit in der Infrastruktur- und Dienstleistungspolitik weiter verliert. Die Debatte um den Einsatz von Bundeswehrsoldaten außerhalb des Bündnisfalles wurde in der GVK auf- 
gegriffen (1993c), aber nicht zum Abschluß gebracht. 5 Außerhalb der GVK schließlich fand die in ihre Schlußphase tretende Debatte über den Freiheitsabbau durch Zulassung des $»$ Großen Lauschangriffes statt. ${ }^{6}$ Die Aufspaltung der Themen und Foren wirkt hier umso nachhaltiger, als die politisch von einem Parteitagsbeschluß inzwischen getragene Zustimmung der SPD zu einer - verfahrensrechtlich gehegten - Zulassung des »Großen Lauschangriffes « entkoppelt wurde von den Bemühungen der Partei, durch Einfügung eines Datenschutzgrundrechtes und die Absicherung der Stellung der Datenschutzbeauftragten insgesamt die Freiheitlichkeit des Grundgesetzes zu stärken.

Die konservative Strategie, eine Gesamtreform zu verweigern, um punktuell und außerhalb des Gesamtrahmens die Freiheitlichkeit des Grundgesetzes abzubauen, setzt sich fort mit den Überlegungen zu Eingriffen in die Tarifautonomie im Rahmen der Diskussion uiber die Finanzierung der Pflegeversicherung (Säcker 1994) und den jüngsten Vorschlag des Fraktionsvorsitzenden der Union, mit der Zulassung von Polizeieinsätzen der Bundeswehr im Inneren einen Kernpunkt des bei der Notstandsgesetzgebung gefundenen Verfassungskonsenses aufzugeben. Ein Blick in den heute geltenden Verfassungstext zeigt: Das Grundgesetz hat im Zuge der Verfassungsreform gegenüber der zum Beitrittszeitpunkt geltenden Fassung an Freiheitlichkeit - so die ernüchternde Zwischenbilanz - nicht gewonnen, sondern verloren.

5 Daß die GVK hier keine Empfehlungen ausgesprochen hat, wird - entgegen der sonst von der Union vertretenen Linie - politisch nicht als Bestätigung des geltenden Verfassungskonsenses verstanden, zumal formell die hierauf bezogenen Gesetzentwürfe und Anträge (BT-Drucks. 12/4107, 4135, 2895, 4534, 3055, 3014) nicht erledigt sind und die Union mit Unterstiutzung aus der wissenschaftlichen Publizistik - versucht, ihren zu Recht kritisierten (s. nur Preuß 1993b) Standpunkt durchzusetzen, es bedürfe lediglich der verfassungsgesetzlichen Klarstellung.

6 Vgl. dazu den Beschluß des SPD-Parteitages in Wiesbaden »Öffentliche Sicherheit und Schutz vor Kriminalität in der Bundesrepublik Deutschland« sowie den am 27.01.1994 der Öffentlichkeit vorgestellten Entwurf eines »Zweiten Gesetzes zur Bekämpfung des illegalen Rauschgifthandels und anderer Erscheinungsformen der Organisierten Kriminalität« (BT-Drs. 12/6784), in den neben einer Änderung des Art. 13 GG zur erleichterten Einziehung von Vermögen, bei dem der Verdacht der Herkunft aus schweren Straftaten nicht ausgeräumt werden kann, die Ergänzung der Eigentumsgarantie des Art. 14 GG gefordert wird. Zur Diskussion siehe auch die Beiträge im Vorgänge-Themenheft »Innere Sicherheit « (Nr. 124, Dezember 1993) und Seifert 1992b. Dieser in der Ausformung sicher verbesserungswürdige und -bedürftige konzeptionelle Vorstoß der SPD auf die wirtschaftliche Machtseite - das Geld - hat in ersten Reaktionen zu einer bislang unbekannten großen Koalition von Bürgerrechtlern und Bankenwelt geführt - mit je ernstzunehmenden rechtsstaatlichen Bedenken. 


\section{Verfahren und Formen der Verfassungsdebatte}

Unabhängig von den in der GVK erzielten Ergebnissen ist ein Ziel der Verfassungsdiskussion schon durch das Verfahren verfehlt worden. Als Selbstverständigung der Bürgerinnen und Bürger des geeinten Deutschlands sollte bereits die gemeinsame Arbeit an den Strukturen der neuen Verfassung integrative Wirkung entfalten (Kuratorium 1991, 11f., 18f.). Der Prozeß sollte offen, transparent und auf Beteiligung angelegt sein und zugleich deutlich machen, daß es sich bei den anstehenden Verfassungsdebatten nicht um Regelverfassungsänderungen, wie sie das Grundgesetz nunmehr schon $40 \mathrm{Mal}$ erfahren hat, handelte. Artikel 5 Einigungsvertrag selbst schon ein Formelkompromiß - legte Form und Verfahren der Verfassungsdiskussion nicht fest und begünstigte so strukturell die Position der Union, welche keinen Neukonstituierungs-, sondern allenfalls geringfügigen Änderungsbedarf sah (vgl. Rüttgers/Oswald 1992; Scholz 1993c). In der Sache wirkt hier die falsche Alternative über den richtigen Weg zur deutschen Einheit - Beitritt nach Art. 23 Satz 2 GG a.F. wider Verfassungsschöpfung nach Art. 146 GG - fort (Grimm 1992, 1059). Der Beitritt nach Art. $23 \mathrm{GG}$ als schnelle und einfache Möglichkeit des staatlichen Zusammenschlusses hindert nicht die Neukonstituierung in Anwendung des Art. 146 GG; und niemand, der auf der Grundlage des Art. 146 GG die Ausarbeitung einer neuen Verfassung anstrebte, wollte in der Sache bewährte Grundsätze aufgeben. Dies konzedieren selbst Konservative wie der ehemalige Bundesverteidigungsminister Scholz, der von der CDU gestellte Vorsitzende der GVK (1993a, 70 f). Der Verfassungsentwurf des Runden Tisches (Fischer 1990; Preuß 1990; Emmerich 1990; Häberle 1990; Thayssen 1990, 143ff.) etwa lehnt sich in seinen Grundstrukturen eng an das Grundgesetz an und enthält nichts, was unter der Geltung des Grundgesetzes nicht auch durch den verfassungsändernden Gesetzgeber beschlossen werden könnte. Gleiches gilt für den vom Kuratorium für einen demokratischen Bund deutscher Länder vorgelegten Verfassungsentwurf (dazu Guggenberger/Preuß/Ullmann 1991; Günther 1991; Seifert 1991; Narr 1991). Die Abwehr der Vorschläge, einen Verfassungsrat einzusetzen, dem nach Vorstellung der SPD nicht nur Inhaber politischer Funktionen, sondern Persönlichkeiten aus allen Bereichen des öffentlichen Lebens und vor allem Persönlichkeiten der Bürgerbewegung in der ehemaligen DDR angehören sollten, spiegelt so schon im Verfahren die Furcht der Konservativen vor einer breiten Verfassungsdebatte und die Notwendigkeit, die Verfassungsbestimmungen einer Legitimitätsprüfung unterziehen zu müssen, aus der die meisten gestärkt hervorgegangen wären. Sie hat zugleich Symbolgehalt: Mit der Festlegung auf das - im Kern unveränderte - Grundgesetz 
wird deutlich gemacht, daß Veränderungswille, Anpassungsleistungen und Umdenken vor allem von der früheren DDR und ihren Bürgerinnen und Bürgern verlangt werden (Grimm 1992, 1060).

\section{Zusammensetzung und Arbeitsweise der GVK}

Die Überweisung der Verfassungsdebatte an die Gemeinsame Verfassungskommission führt die Debatte näher an das Regelverfahren der bloBen Verfassungsänderung ohne besondere Bedeutung heran. Die GVK bestand aus je 32 Vertreterinnen und Vertretern von Bundestag und Bundesrat (GVK 1993a, 120 ff). Sie war also ausschließlich mit Politikerinnen und Politikern besetzt, die auch sonst mit Gesetzgebungs- und Änderungsaufgaben betraut sind. Den besonderen Änderungsanstößen wurde durch die Besetzung nicht Rechnung getragen: Lediglich fünf der 32 Bundestagsabgeordneten stammten aus den ostdeutschen Ländern, vier der zehn ostdeutschen Ländervertreter waren »Westimporte« (Lieberam/ Heuer 1993). Die Besetzung mit parteigebundenen Politikerinnen und Politikern prägte nicht nur den Verlauf der Sachdiskussion vor, wenngleich sich bei Themen mit spezifischem Länderbezug Überlagerungen der Parteifronten durch die Bund-Länder-Problematik ergaben. ${ }^{7}$ Die »Professionalisierung « der Verfassungsdebatte und ihre Einbindung in den Alltagsbetrieb Bonner Politikproduktion begünstigte eine Überlagerung durch tagespolitische Interessen und Wahrnehmungen und erschwerte, die politischen Parteien selbst, die Konstitutionalisierung und Hegung ihrer politischen Macht und ihren Beitrag zur Politikverdrossenheit, zum Gegenstand der Reformüberlegungen zu machen (Grimm 1992, 1060f.; Benz 1993, 884). Eine Begrenzung der politischen Parteien und ihres Einflusses auf Staat und Gesellschaft, eine Beschneidung des »expandierenden Parteienstaats« (Grimm 1994) kann von den Vertreterinnen und Vertretern gerade jener Parteien, deren Vermittlungsfunktion zwischen Volk und Staat merklich nachläßt, nur bedingt erwartet werden.

Ein Argument für eine $»$ Politikerkommission« war, daß bei einem politisch nicht eingebundenen und durch Wahlakt formell legitimierten Gremium die Gefahr bestanden hätte, daß nach intensiveren, intellektuell möglicherweise noch qualifizierteren und jedenfalls von der Tagespolitik entkoppel-

7 Bei den Ländern mit Koahitionsregierungen (z.B. Berlin, Rheinland-Pfalz und BadenWürttemberg) ist es - anders als es Art. 51 Abs. 3 Satz 2 GG für die Stimmabgabe im Bundesrat vorschreibt - zu gespaltenem Abstimmungsverhalten gekommen. Die Protokolle der GVK weisen aber nur das Abstimmungsergebnis, nicht das jeweilige Stimmverhalten aus, so daß für Nichtbeteiligte dessen Zustandekommen - gerade auch bei knappen Mehrheiten - nicht zu rekonstruieren ist. Zu namentlichen Abstimmungen ist es nicht gekommen. 
ten Beratungen gefundene Ergebnisse das Schicksal vieler Sachverständigenkommissionen geteilt hätten: wissenschaftliche Anerkennung, aber politische Folgenlosigkeit. Dies traf schon im Ansatz nicht zu. Ein vom politischen Alltagsgeschäft entkoppelter Verfassungsrat hätte nicht im politischen Elfenbeinturm tagen können. Auch er hätte - schon um der Konsensfunktion der Verfassung willen - Durchsetzungsmöglichkeiten in den Blick nehmen und den Kompromiß suchen müssen. Relative politische Unabhängigkeit oder größere politische Freiheit ist nicht gleichzusetzen mit politischer Blindheit. Von der Qualität der Empfehlungen und ihrer Begründung wäre es abhängig gewesen, ob die entscheidenden politischen Instanzen sie hätten übergehen können.

Daß die Besetzung mit aktiven Politikerinnen und Politikern trotz Einbindung in Partei und Fraktion nicht die im wesentlichen unveränderte Übernahme der Empfehlung sicherstellt, zeigen die Schwierigkeiten, die in der Unionsfraktion zu überwinden waren, um selbst die mit der vereinbarten Zwei-Drittel-Mehrheit verabschiedete Empfehlung der GVK in das förmliche Gesetzgebungsverfahren einzubringen. Verfassungsrechtlich ist unbestritten, daß die im Grundgesetz so nicht vorgesehene Gemeinsame Verfassungskommission nur Empfehlungen geben, keine bindenden Gesetzesbeschlüsse fassen kann. Die Bereitschaft und die Fähigkeit der Kommission, sich ein Stïck weit aus dem Routinebetrieb zu lösen und doch in einigen grundlegenden Fragen des Gemeinwesens auf Empfehlungen einigen zu können, ist als positives Zeichen für die politische Kultur im Lande zu werten. Dies bewirkt aber eine erhebliche politische Bindungswirkung. Die Überlegungen in der Union, bei der Umsetzung selbst der im einstimmig verabschiedeten Bericht der Kommission enthaltenen Empfehlungen die Diskussions- und Konsensbildungsprozesse in Frage zu stellen und zu wiederholen, setzte dagegen ein Negativsymbol und würde die Arbeit der Kommission nachhaltiger entwerten, als es die Nichtübernahme der Ergebnisse politisch nicht eingebundener Sachverständigenkommissionen bedeutete. In Zeiten von Ausländerfeindlichkeit und Fremdenhaß ist insbesondere kaum verständlich, daß die Union in der Ersten Beratung der Gesetzentwürfe im Bundestag Anfang Februar 1994 klar erklärt hat, daß sie das von der Kommisison mit 2/3-Mehrheit - also auch Stimmen aus der Union empfohlene und im interfraktionellen Gesetzentwurf enthaltene Staatsziel, das die staatliche Gewalt auf die "Achtung « der Identität ethnischer, kultureller und sprachlicher Minderheiten unabhängig von der Staatsangehörigkeit verpflichten soll, nicht mehr mitträgt. Die Union will nun allenfalls einem Minderheitenschutz zustimmen, der sich auf die traditionell in geschlossenen Siedlungsgebieten ansässigen nationalen Minderheiten deutscher Staatsangehörigkeit beschränkt - also für Dänen, Friesen und Sorben. 
Dies schließt die Sinti und Roma deutscher Staatsangehörigkeit ebenso aus wie verhindert wird, daß deutsche Staatsangehörige etwa türkischer Herkunft sich zur Minderheit in Deutschland zusammenfinden können.

\section{GVK und Öffentlichkeit}

Die GVK und mit ihr das Verfassungsreformthema standen nicht im Zentrum öffentlicher Aufmerksamkeit. Gleichwohl war sie weit mehr als ein im Geheimen tagender »Ausschuß der politischen Elite (Benz 1993, 883). Die Sitzungen und Anhörungen der GVK waren seit der 4. Sitzung öffentlich. Als Gewinn für die Verfassungskultur ist das lebhafte Interesse der Bürgerinnen und Bürger an der Arbeit der Kommission hervorzuheben. Aus der Mitte der Bevölkerung erreichten die Kommission etwa 800.000 Eingaben, die zumeist konkrete Reformvorschläge unterstützten. Für eine unmittelbare Bürgerbeteiligung sprachen sich über 250.000 Eingaben aus, knapp 150.000 Eingaben für den Tierschutz. In diesem Umfange und in dieser Intensität war die Tätigkeit keines vergleichbaren Gremiums Gegenstand von Bürgereingaben und Verbandszuschriften. Gemessen an der Zahl der Eingaben war das Interesse an der Arbeit der GVK sogar weit größer als die Aufmerksamkeit, die die Bevölkerung in den ostdeutschen Ländern ihrer Landesverfassunggebung widmete. Allerdings hat kein »umfassender Dialog mit der Bevölkerung « (Heitmann 1993, 12) stattgefunden; die GVK war auch nicht getragen von einer breiten politischen »Massen«-bewegung. Wer dies verlangt und zum Maßstab macht, überspannt das Interesse, das in der Bevölkerung für Verfassungsfragen zu erwarten und von ihr zu verlangen ist. Dies gilt allzumal für eine Verfassungsdebatte in einem Gremium, das nur geringe Resonanz in den Medien gefunden hat (Benz 1993, 884f.) und in dem es um eine Rejustierung der Verfassungsordnung geht, nicht um eine auch in der Sache grundlegende inhaltliche Neugestaltung, bei der existentielle Fragen mit tagespolitisch spürbarer Wirkung, »Messerund Gabel-Fragen « im Sinne Seiferts $(1993,91)$, zu entscheiden gewesen wären. Die Zuschriften sind indes nicht ohne jeden Einfluß geblieben: Sie waren einer der Faktoren, die für die Staatsziele Umweltschutz und Frauenförderung die zunächst nicht bestehende Zwei-Drittel-Mehrheit für eine Empfehlung gesichert haben. Die Transparenz der Arbeit der GVK hatte allerdings ihre Grenze bei den Prozessen der Entscheidungs- und Kompromißbildung selbst: Sie waren überwiegend in das Arkanum der Obleuteund vor allem Berichterstattergespräche sowie sonstiger informeller Vorbereitungs- und Koordinationsgremien verlagert (GVK 1993a, 10f.). 


\section{Quorum als Sellbstbeschränkung oder Sellbstaufgabe der Verfassungsdebatte?}

Die Ergebnisse der GVK sind wesentlich von der frühzeitigen Festlegung darauf geprägt, daß für Empfehlungen eine Zwei-Drittel-Mehrheit erforderlich sei. Dies entspricht dem Quorum, das Art. 79 GG für den Gesetzesbeschluß über Verfassungsänderungen erfordert, und ist so immanent stimmige Konsequenz des Heranrückens der Verfassungsdiskussion an das Regelgesetzgebungsverfahren. Dieses Quorum verstärkt aber die durch die Besetzung vorgegebene Orientierung der Diskussions- und Entscheidungsabläufe am Kriterium der Durchsetzbarkeit im parlamentarischen Verfahren und minderte die Chancen einer innovativen, offenen Debatte (Benz 1993, 885; Berlit 1993a, 89).

Für die Entscheidungsfindungsprozesse bekamen so die unterschiedlichen Interessen an einer Verfassungsreform tragendes Gewicht. Nach ihrer Idee und den Befürwortern einer Verfassungsreform sollte die GVK ein Gremium sein, dessen Verhandlungen dem Diskursmodell folgen und in dem allein die besseren Argumente unter Rückgriff auf die allgemein anerkannten Prinzipien der Gestaltung der grundgesetzlichen Ordnung zählen sollten; hier herrschte das Modell einer »prinzipiengeleiteten Argumentation über Grundstrukturen der Verfassungsordnung « (Benz 1993, 887) vor. Die institutionelle Anbindung und Ausgestaltung der GVK dagegen begünstigte den Rückgriff auf die auch das politische Alltagsgeschäft bestimmenden Tausch- und $\gg$ bargaining"-Strukturen. Einem unbegrenzten $\gg$ Feilschen um Verfassungsnormen « widerstritt verfassungspolitisch allerdings bereits die Dignität der Verfassung. Auch geht es bei der Verfassung um die Festlegung von Grundsätzen und Prinzipien, die sich weniger für Tauschhandeln eignen und thematisch nicht hinreichend zusammenhängen: Politisch wäre etwa nicht vermittelbar gewesen, die Zustimmung zu einer Verfassungsänderung im Interesse der Privatisierung der Luftverkehrsverwaltung von der Zustimmung der Einführung sozialer Staatszielbestimmungen abhängig zu machen. Vor allem aber gab es kein gemeinsames Interesse an einer breit angelegten Verfassungsreform in allen Parteien. Die konservative Mehrheit im Deutschen Bundestag sah im Kern keinen Änderungsbedarf. Aus ihrer Sicht bedeutete der Beitritt der DDR den Eintritt in eine nicht reformbedürftige, bewährte und stabile Verfassungsordnung, um eine bloße Erweiterung des Verfassungsgebietes. Bereits die Verfassungsdebatte selbst wurde als Gefährdung des Verfassungskonsenses beargwöhnt, der noch in den Feiern zum 40jährigen Bestehen des Grundgesetzes hervorgehoben war, die zur Kompensation wiedervereinigungsbedingter Frustration durch Verfassungsaktionismus künstlich herbeigeredet und nur 
noch psychologisch erklärbar sei (so prononciert Isensee 1992a; 1992b; 1993; mit dem Begriff »Verfassungsschwärmerei« sanfter Merten 1992). Das Grundgesetz als die »beste Verfassung, die Deutschland je hatte«, sollte in Struktur und im Detail unangetastet bleiben, um seine Stabilität zu wahren. Hinter all dem stand die - unberechtigte - Sorge, die Befürworter einer Verfassungsreform wollten eine auch inhaltlich umstürzende Verfassungsreform, verfolgten einen »postsozialistischen Traum, über eine neue gesamtdeutsche Verfassung eine andere Republik heraufzuführen« (Isensee 1993, 2584; Hennis 1993, 35).

\section{Ergebnisse der GVK als Bestätigung des GG?}

Unter diesen Bedingungen ist es politisch verständlich, aber zumindest verkürzend, als ein wesentliches und positiv zu wertendes Ergebnis der GVK hervorzuheben, daß das Grundgesetz im wesentlichen unverändert geblieben und hieraus eine Bestätigung des Verfassungskonsenses insgesamt herzuleiten ist (so etwa Scholz 1993b, 4; Isensee 1993; Heitmann 1993; 1994). Konnte für ein Veränderungsbegehren die erforderliche ZweiDrittel-Mehrheit nicht erzielt werden, bedeutete dies lediglich, daß ein neuer Verfassungskonsens für die geänderte Verfassung nicht hat gefunden werden können. Eine positive politische Bestätigung der rechtlich unverändert fortgeltenden Verfassungslage bedeutet dies namentlich dann nicht, wenn sich für eine Änderung einfache oder gar deutliche absolute Mehrheiten, aber eben nicht die Zwei-Drittel-Mehrheit haben finden lassen. Die Logik dieser defensiven Beharrungsargumentation erschließt sich nur, wenn der durch die deutsche Einigung bewirkte qualitative Sprung ausgeblendet, Legitimationsschwächen des Grundgesetzes verneint, oder doch zumindest durch eine »Legitimation durch Erfolg « (Storost 1991) als zwischenzeitlich überwunden gewertet werden (Isensee 1992; Würtenberger 1993, 747) und eine Partizipation der ostdeutschen Bürgerinnen und Bürger an der Gestaltung der Verfassungsordnung nicht für erforderlich gehalten werden.

Die Abwehr von Veränderungen als Sieg für die Stabilität und Kontinuität der Verfassung auszugeben, bedeutet nicht nur, eine »self-fulfilling prophecy« als Legitimationsstütze zu mißbrauchen. Das Argument hat auch eine machtpolitische Dimension. Die Betonung der abwehrrechtlichen Gehalte der Grundrechtsordnung und seiner Offenheit im iibrigen stabilisiert in der Sache die bestehenden sozio-ökonomischen Verhältnisse, schirmt sie gegen Kritik ab und blendet die Anforderungen aus, die sozialer Schutz und Ausgleich für die sozial Schwachen an die Verfassung stellen. Freiheitsrechte sichern nicht die Voraussetzungen des Freiheitsgebrauches. Der 
moderne Sozialstaat des Grundgesetzes hat die Sicherung der existentiellen Grundbedürfnisse der Menschen umfassend in den Blick zu nehmen und durch staatliche Vorsorge und Leistungen sicherzustellen. Diese Aufgabe findet im allgemeinen Sozialstaatsprinzip des Grundgesetzes keinen hinreichenden Niederschlag. Wenn die auf Veränderungsabwehr gerichtete Position die formale Struktur des Grundgesetzes als »justiziabler Rechtsverfassung «, als nüchternes Verfassungsgesetz mit kargen aber klaren Rahmenvorgaben für einen offenen und wegen seiner Offenheit erfolgreichen politischen Prozeß betont, ist dies mehr als eine scheinbar neutrale, verfassungsdogmatische Position: Es bedeutet ein »weiter-so-Denken« unter Leugnung inhaltlichen Veränderungsbedarfs, der dahinterstehenden Probleme und ein Festhalten an den bestehenden Machtverhältnissen.

\section{Eine demokratische Linewerung des Grundgesetzes findet nicht stati}

Die Verfassungsdebatte zerfiel in viele Einzelthemen unterschiedlicher Politikfelder, in denen sich zusätzlich zu den divergierenden Verfassungskonzeptionen die feldbezogenen Politikunterschiede auswirkten. Von den vielen Einzelthemen und Ergebnissen soll hier das Unvermögen der GVK herausgegriffen werden, sich trotz Politikverdrossenheit und Parteienfrust auf eine Stärkung des demokratischen Gehalts des Grundgesetzes zu einigen. Selbst vorsichtige Ansätze eines Mehrs an bürgerschaftlicher Mitwirkung sind an der Blockadehaltung der Union gescheitert. Gegen die Aufnahme direktdemokratischer Elemente in das Grundgesetz wurden die altbekannten, durch ständige Wiederholung nicht überzeugenderen Grïnde ins Feld geführt: Direkte Demokratie schwäche das erfolgreiche repräsentative System, lasse sachgerechte Lösungen der immer komplexer werdenden Probleme nicht erwarten, überfordere die Bevölkerung, öffne populistischen, gefühlsgeleiteten Kampagnen Tür und Tor, sei minderheitenfeindlich und verschiebe das föderative Zusammenspiel von zentralstaatlicher und gliedstaatlicher Demokratie in allzu zentralistische Richtung, weil das Bundesvolk in seiner Gesamtheit entscheide (zur Diskussion Mayer 1993; Berlit 1993b; Preuß 1993a; Luthardt 1993). Die erfolglos vorgeschlagenen Grundgesetzänderungen tragen durch eine sorgsame Ausgestaltung all diesen Bedenken, die von einem diffusen MiBtrauen in die politische Reife des eigenen Volkes und von einer obrigkeitsstaatlich geprägten Überschätzung repräsentativer Politikeliten getragen sind, Rechnung. Der Blick in die Länder (G. Jürgens 1993; Jung 1993; Hoof/Kempf 1993) und auf das Ausland (Butler/Ranney 1980; Bugiel 1987; Strübel 1991; Möckli 1991) macht deutlich, daß Elemente direkter Demokratie als Ergänzung des repräsentativen Systems dieses stabilisieren, in seinen Wirkungs- und Lei- 
stungsvoraussetzungen fördern, Wahrnehmungs- und Verarbeitungssperren des Politikbetriebes überwinden helfen können und sie vor allem als Form einer »Selbstorganisation von Gesellschaft« Möglichkeit bieten, Mitverantwortung und Engagement für das Gemeinwesen zu beweisen. Direktdemokratische Elemente hätten vor allem auch eine gewisse Begrenzung der Politikformulierungs- und Gestaltungsmacht der politischen Parteien bedeutet (Grimm 1992, 1065), die allein schon durch die bloße Möglichkeit direktdemokratischer Einwirkung und Kontrolle weiter hätten sensibilisiert und dazu angehalten werden können, gesellschaftlichen Problemen von hoher Relevanz nicht auszuweichen.

Auf der Verlustliste der Bemühungen um mehr demokratische Teilhabe in allen Fragen von Staat und Gesellschaft stehen weiterhin die Vorschläge zu einer Erweiterung des Wahlrechts auf Ausländerinnen und Ausländer auch über den EG-Bereich hinaus, eine Änderung des Bürgerinnen- und Bürgerbegriffs des Grundgesetzes, der Ausländerinnen und Ausländern nach einer gewissen Aufenthaltsdauer den Bürgerstatus auch ohne Wechsel der Staatsangehörigkeit öffnet, Überlegungen zur Demokratisierung von Institutionen, die von den Gewerkschaften verlangte verfassungsförmige Absicherung betrieblicher und überbetrieblicher Mitbestimmung (DGB 1992) sowie jene Vorschläge zum Parlamentsrecht, die die Informations- und Kontrollrechte einzelner Abgeordneter und der Oppositionsfraktionen im Interesse von mehr Transparenz und öffentlichkeit verstärken wollten. Als mit Recht nicht durchsetzungsfähig hat sich dagegen der Vorstoß von Bündnis 90/Grüne erwiesen, Bundespräsidentin oder Bundespräsident direkt vom Volk wählen zu lassen: Das politische Gewicht der direkten demokratischen Legitimation hätte in einem unauflöslichen Widerspruch zu den Aufgaben und Befugnissen des Amts gestanden. Es machte Sinn nur in einem von keiner Partei gewollten Übergang zu einem Präsidialsystem amerikanischen oder französischen Vorbilds.

\section{Keine bessere Verfassung}

Das breite Feld der von der GVK erörterten Reformanliegen und die Gesamtzahl der empfohlenen Änderungen weisen einige Aktivposten auf. Trotz des deutlichen Kompromißcharakters sind hier als Beispiele zu nennen die Staatsziele Gleichberechtigung der Frauen, Umweltschutz und Minderheitenschutz, die viele Wünsche offen lassen, aber einen Schritt in die richtige Richtung bedeuten, ohne die Entwicklung zu begrenzen. Bei einer Bilanz überwiegt indes eindeutig der Negativsaldo. Weit davon entfernt, eine neue Verfassung oder ein wesentlich geändertes Grundgesetz zu sein, ist in der Reformdebatte die Chance verpaßt worden, das Grundgesetz 
problemgerecht fortzuentwickeln und die in Verfahren und Inhalten einer Verfassungsreform angelegten Integrations- und Legitimationschancen zu nutzen. Die Enttäuschung spiegeln Kommentarüberschriften wider wie z.B. »Außer Spesen nichts gewesen? (Incesu 1993a), »Verspielte Chancen« (Incesu 1993b), »Die gescheiterte Erneuerung des Grundgesetzes« (Seifert 1993) oder »Das Grundgesetz - auf Grund gesetzt« (Schneider 1994).

Gleichwohl hat die SPD auf ihrem Wiesbadener Parteitageinstimmig beschlossen, zentrale Reformvorstellungen weiterzuverfolgen. Ihre Durchsetzungschancen sind angesichts wachsender Widerstände in der Union, die teils von einer gemeinsamen Stellungnahme verschiedener Arbeitgeberverbände (BDA, BDI, DIHT, ZDH) und der verfassungsrechtlichen Publizistik (Kriele 1993) unterstützt werden, gegen eine Übernahme selbst der in der GVK gefundenen Kompromisse gering. Wenig spricht auch dafür, daß sich mit Beginn der förmlichen parlamentarischen Beratungen das Medieninteresse an Fragen der Verfassungsreform nachhaltig erhöhen wird. Unter den Bedingungen einer »Mediendemokratie « fällt es schwer, Fragen der Verfassungsreform in ihrer Gesamtheit zum Gegenstand breiten politischen Interesses werden zu lassen - ungeachtet ihrer langfristigen Bedeutung. Dies gilt um so mehr als der Verfassungsbezug der drängendsten Probleme wie Massenarbeitslosigkeit und Wirtschaftsentwicklung nur vermittelt ist. Im Bereich der Besetzung von Verfassungsthemen hat sich zudem - nicht zuletzt auch mit Unterstiitzung ihr zugeneigter Medien - die Union bislang als erfolgreicher erwiesen, wie als jüngstes Beispiel der Vorstoß des Fraktionsvorsitzenden der CDU/CSU-Bundestagsfraktion Schäuble kurz vor Weihnachten 1993 zugunsten eines Polizeieinsatzes der Bundeswehr im Inneren zeigt. Die Union hat es indes auch bei den Debatten um eine Änderung des Grundgesetzes im Bereich des Asylrechts, des Auslandseinsatzes von Bundeswehrsoldaten und des $»$ Großen Lauschangriffs « geschickter als die SPD verstanden, ihre problemverkürzende und -verzerrende Sicht in den Medien zu verankern. Ihr kommt dabei zugute, daß die Vermittlung komplexer Zusammenhänge - etwa von Bundeswehreinsatz im Ausland, Friedensstaatlichkeit des Grundgesetzes und des Selbstverständnisses von der Rolle Deutschlands in der Welt - immer schwieriger ist als die Verkündigung scheinbar einfacher Wahrheiten. Die konservative Strategie einer schrittweisen Befreiung ihrer Machtpolitik aus den Fesseln und Bindungen der Verfassung baut dabei auf jenen Mechanismen einer politischen $\gg$ Stimmungsdemokratie « auf, welche die Konservativen als vermeintliche Gefahr von Elementen direkter Demokratie behaupten.

Ob die SPD demgegenüber Alternativen hatte, sie sich ohne politische Not hat in die Defensive drängen lassen oder gar ohne innere Überzeugung von der Richtigkeit freiheitsabbauenden Grundgesetzänderungen zugestimmt 
hat, um eine Konzentration der politischen Debatte auf die für sie zentralen Fragen von Arbeitslosigkeit und Sozialabbau zu ermöglichen, kann hier nicht erörtert oder entschieden werden. Die Schwierigkeiten der Vermittlung eines von tagespolitischen Aktualitäten befreiten Verfassungsdiskurses unter Bedingungen, in denen Politik und Öffentlichkeit nur eine begrenzte Anzahl von Themen wahrnehmen und verarbeiten können, mag das Beispiel der Behandlung der Empfehlungen der GVK in der Bundesratssitzung vom 17. Dezember 1993 zeigen: Der von allen Ländern unterstützte Beschluß des Bundesrates, die Empfehlungen der GVK als Gesetzentwurf einzubringen, wurde in den Medien kaum wahrgenommen, weil am selben Tage der Bundesrat über die Pflegeversicherung, die Bahnreform und das Haushaltsgesetz mit allen Begleitgesetzen Beschluß gefaßt hat. Für eine der grundlegenden Bedeutung des Themas angemessene Berichterstattung über die Verfassungsreformfragen war neben diesen Themen kein Raum mehr.

Unter dem Aspekt der Vermittelbarkeit der Verfassungsreformdebatte begegnet es gewissen Zweifeln, ob die Themenbreite und -vielfalt der Verfassungsthemen, die nach dem Beschluß des SPD-Parteitages in Wiesbaden weiterzuverfolgen sind, sinnvoll gewählt war. Unbestritten ist, daß all die genannten Themen zentrale und wichtige Reformanliegen sind. Die in der GVK von der Union gezeigte strikte Verweigerungshaltung spricht letztlich aber dafür, daß die Vielfalt der aufgegriffenen Themen die Durchsetzungschancen notwendiger Änderungen des Grundgesetzes nicht noch zusätzlich gemindert hat. Nützlich wäre die Konzentration wohl nur dann, wenn die Union sich offener und beweglicher gezeigt hätte.

Der Verzicht selbst auf die notwendige Erneuerung der Verfassung läßt langfristig die Schwächung seiner Fähigkeiten befürchten, einen stabilen Ordnungsrahmen für Staat und Gesellschaft und zur Regulierung von Politik zu bieten. Daß sich nun das Scheitern sachlich begründeter Grundgesetzänderungen, die die Bezeichnung »Verfassungsreform « oder Modernisierung des Grundgesetzes verdiente, an den politischen Machtverhältnissen abzeichnet, ändert nichts an dem objektiven Problemdruck und den sachlichen Änderungsnotwendigkeiten. Auch wenn sie von (zu) großen Hoffnungen getragen gewesen sein mag, es Fehler gab und die politischen Kräfteverhältnisse nicht klar genug analysiert worden sein mögen: Dem Grunde nach gab es zur Verfassungsdiskussion aus Anlaß der staatlichen Einheit keine Alternative. Ein Verfassungsdiskurs kann Politik nicht ersetzen und muß selbst von einer politischen Bewegung getragen sein: Zur Regulierung und Bändigung von Politik durch Verfassung gibt es indes keine Alternative. Der Kampf um Verfassungspositionen und das Streben nach einer Verfassung, die Freiheit und Menschenrechte unter den gewandelten 
Bedingungen zeitgemäßer zu wahren und zu entfalten hilft, bleibt so auch künftig Aufgabe.

\section{Literatur}

Asmussen, Claus/ Eggeling, Ulrich 1993: Empfehlungen des Bundesrates zur Stärkung des Föderalismus in Deutschland und Europa, Verwaltungsarchiv 84 (1993), 230-259.

Bartlsperger, Richard 1990: Verfassung und verfassunggebende Gewalt im vereinten Deutschland, DVBI. 1990, 1285-1301.

Benz, Arthur 1993: Verfassungsreform als politischer Prozeß, DÖV 1993, 881-889.

Berghahn, Sabine 1993: Ehe und Familie in der Verfassungsdiskussion - vom institutionellen zum sozialen Grundrechtsverständnis?, KJ 1993, 397-418

Berlit, Uwe 1993a: Modifizierung oder Modernisierung? Zur Verfassungsdiskussion nach der staatlichen Einigung Deutschlands, $G M H$ 1993, 76-88

Berlit, Uwe 1993b: Soll das Volk abstimmen? Zur Debatte über direktdemokratische Elemente im Grundgesetz, KritV 1993, 318-359

Blumenwitz, Dieter 1991: Wie offen ist die Verfassungsfrage nach der Herstellung der staatlichen Einheit Deutschlands?, APuZ B 49/1991, 3-11.

Blumenwitz, Dieter 1992: Braucht Deutschland ein neues Grundgesetz?, ZfP 39 (1992), 1-23

Bock, Bettina 1990: Umweltschutz im Spiegel von Verfassungsrecht und Verfassungspolitik, Berlin 1990.

Böckenförde, Ernst Wolfgang 1991: Die sozialen Grundrechte im Verfassungsgefüge, in: ders.: Staat, Verfassung, Demokratie, 146-158.

Bruns, Manfred 1993: Homosexuelle im Recht: Toleriert, aber nicht akzeptiert, Betrifft JUSTIZ Nr. 34, 1993, 82-86.

Bugiel, Karsten 1987: Das Institut der Volksabstimmung im moderenn Verfassungsstaat. Zur Verfassungsrechtslage und Rechtspraxis bürgerlicher Sachentscheidungsrechte, ZParl 1987, 394-419.

Bumke, Ulrike 1993: Art. 3 GG in der deutschen Verfassungsdiskussion, Der Staat 32 (1993), 117-139.

Butler, David/Ranney, Austin (Hrsg.) 1980: Referendums, 2. Aufl. Washington 1980.

Deter, Gerhard 1993: Frauenrechte in den Verfassungsentwürfen der neuen Bundesländer, ZRP 1993, 22-29.

DGB 1992: Alle Staatsgewalt geht vom Volke aus. Verfassungsdebatte in Deutschland - Leitlinien und Argumente des DGB, Düsseldorf 1992.

Eicher, Hermann 1988: Der Machtverlust der Landesparlamente, Berlin 1988.

Emmerich, Klaus 1990: Geschichte des Verfassungsentwurfs des Runden Tisches, DuR 1990, 376-381.

Enquête-Kommission Verfassungsreform 1972: Zwischenbericht der Enquete-Kommission Fragen der Verfassungsreform zum Ende der 6. Legislaturperiode des Deutschen Bundestages, BT-Drs. 6/3829.

Enquête-Kommission Verfassungsreform 1976: Schlußbericht der Enquete-Kommission Fragen der Verfassungsreform, BT-Drs. 7/5924 (9.12.1976).

FES 1991: Deutschland in neuer Verfassung. Verfassungspolitischer Kongreß der FriedrichEbert-Stiftung 13.-15. Juli 1990 in Potsdam, 15.-16. März 1991 in Bonn, Bonn 1991

Fischer, Erich (Hg.) 1990: Verfassungen der DDR, Baden-Baden 1990.

Fischer, Wolfgang 1993: Die Europäische Union im Grundgesetz: der neue Artikel 23, ZParl. 1993, 32-49.

Franke, Dietrich/ Hofmann, Rainer 1993: Nationale Minderheiten - ein Thema für das Grundgesetz?, EuGRZ 1992, 401-409.

Frowein, Jochen Abr./Hofmann, Rainer/Oeter, Stefan (Hg.) 1993: Das Minderheitenrecht europäischer Staaten, Teil 1, Berlin u.a. 1993.

Gerhard, Ute 1991: Frauen für eine neue Verfassung, Vorgänge 110 (2/1991), 63-71. 
Grawert, Rolf 1979: Zur Verfassungsreform, Der Staat 18 (1979), 229-258.

Grimm, Dieter 1990b: Die Zukunft der Verfassung, Staatswissenschaft u. Staatspraxis 1990, 4-33.

Grimm, Dieter 1992: Verfassungsreform in falscher Hand?, Merkur 1992, 1059-1072.

Grimm, Dieter 1994: Politische Parteien, in: Ernst Benda/Werner Maihofer/Hans-Jochen Vogel (Hrsg.), Handbuch des Verfassungsrechts, 2. Aufl., 1994 (i.E.), \$ 14

Grimm, Dieter (Hg.) 1990a: Wachsende Staatsaufgaben - sinkende Steuerungsfähigkeit des Rechts, Baden-Baden 1990.

Guggenberger, Bernd/Preuß, Ulrich K./Ullmann, Wolfgang 1991: Eine Verfassung fiir Deutschland, München/ Wien 1991.

Guggenberger, Bernd/Stein, Tine (Hg.) 1991: Die Verfassungsdiskusssion im Jahr der deutschen Einheit, München 1991.

Günther, Uwe 1991: Verfassungsreform, Verfassungslyrik und Politik. Anmerkungen zum Verfassungsentwurf des »Kuratoriums für einen demokratisch verfaßten Bund deutscher Länder «, KJ 1991,369-374.

GVK 1992: Stenographisches Protokoll der 1. Öfentlichen Anhörung der Gemeinsamen Verfassungskommission »Grundgesetz und Europa, insbesondere Artikel 24, 28, 32, 88109 $\mathrm{GG} \ll$ vom 22. Mai 1992

GVK 1993a: Gemeinsame Verfassungskommission von Bundestag und Bundesrat. Bericht gemäß Beschluß des Deutschen Bundestages - Drucksachen 12/1590, 12/1670- und Beschluß des Bundesrates - Drucksache 741/91 (Beschluß), BT-Drucks. 12/6000 vom 5.11 .1993

GVK 1993b: Stenographisches Protokoll der 8. Öffentlichen Anhörung der Gemeinsamen Verfassungskommission (zugleich 55. Sitzung des Innenausschusses und 71. Sitzung des Rechtsausschusses) zu den Gesetzentwürfen zur Änderung des Asylrechts am 11. März 1993

GVK 1993c: Stenographisches Protokoll der 7. öffentlichen Anhörung der Gemeinsamen Verfassungskommission »Staatliche Souveränität und militärische Verteidigung vom 11.2.1993

GVK 1993d: Stenographisches Protokoll der 7. öffentlichen Anhörung der Gemeinsamen Verfassungskommission "Staatliche Souveränität und militärische Verteidigung« vom 11.2 .1993$.

Hailbronner, Kay 1993: Die Asylrechtsreform im Grundgesetz, 107-117.

Häberle, Peter 1990: Der Entwurf der Arbeitsgruppe »Neue Verfassung der DDR $«$ des Rundes Tisches (1990), JöR N.F. 39 (1990), 319-349.

Häberle, Peter 1991/92: Das Problem des Kulturstaates im Prozeß der deutschen Einigung Defizite, Versäumnisse, Chancen, Aufgaben, JöR N.F. 40 (1991/92), 291-365.

Häberle, Peter 1992a: Die Kontroverse um die Reform des Grundgesetzes (1991/92), Zeitschrift für Politik 1992, 233-263.

Häberle, Peter 1992b: Die Verfassungsbewegung in den fünf neuen Bundesländern, $J \ddot{o R}$ N.F. 41 (1992), 69-92.

Heitmann, Steffen 1993: Etwas hat sich geändert - vieles wird sich ändern. Das Grundgesetz bleibt - Gedanken zum Abschluß der Arbeit der Gemeinsamen Verfassungskommission, FAZ 22.11.1993, 12.

Heitmann, Steffen 1994: Die mißbrauchte Einheit. Bemerkungen zur Verfassungsdiskussion im wiedervereinten Deutschland, in: Klaus Letzgus (Hg.): Für Recht und Staat, München 1994, 217-227.

Hennis, Wilhelm 1993: Auf dem Weg in eine ganz andere Republik. Die geplante Verfassungsreform verschiebt die Statik des Grundgesetzes, FAZ 26.02.1993, 35.

Herdegen, Matthias 1991: Die Verfassungsänderungen im Einigungsvertrag, Heidelberg.

Herdegen, Matthias 1992: Die Aufnahme besonderer Vorschriften zugunsten behinderter Personen in das Grundgesetz, VSSR 1992, 245-263.

Herdegen, Matthias 1993: Die Aufnahme besonderer Rechte des Kindes in die Verfassung, FamRZ 1993, 374-384.

Heuer, Uwe-Jens/Riege, Gerhard (Hg.) 1992: Neues Deutschland. Neue Verfassung?!, Bonn. 
Hofmann, Hans 1993: Grundgesetz und Europäische Union. Fortentwicklung der Verfassung im Zuge des europäischen Einigungsprozesses, APuZ B 52-53/93, 33-39.

Hofmann, Rainer 1992: Minderheitenschutz in Europa. Überblick über völker- und staatsrechtliche Lage, ZaöRV 52 (1992), 1-69.

Hoof, Claus/Kempf, Antonio 1993: Dokumentation zur plebiszitären Praxis und Verfassungsrechtslage in den Bundesländern, ZParl 1993, 14-21.

Incesu, Lotte 1993a: Außer Spesen nichts gewesen? Bilanz der Arbeit der Gemeinsamen Verfassungskommission, Vorgänge 124 (4/1993), 7-14.

Incesu, Lotte 1993b: Verspielte Chancen. Die Arbeit der Gemeinsamen Verfassungskommission, $K J$ 1993, 475-489.

Isensee, Josef 1990: Staatseinheit und Verfassungskontinuität. 2. Bericht zur Sondertagung der Vereinigung der Deutschen Staatsrechtslehrer »Deutschlands aktuelle Verfassungslage $\ll$, VVDStRL 49 (1990), 39-69.

Isensee, Josef 1992a: Braucht Deutschland eine neue Verfassung?, Köln 1992.

Isensee, Josef 1992b: Die künstlich herbeigeredete Verfassungsdebatte, Die politische Meinung 269 (April 1992), 11-16.

Isensee, Josef 1993: Mit blanem Auge davongekommen - das Grundgesetz. Zu Arbeit und Resultaten der Gemeinsamen Verfassungskommission, NJW 1993, 2583-2587.

Jung, Otmar 1993: Daten zu Volksentscheiden in Deutschland auf Landesebene (1946-1992), 1993, 5-13.

Jürgens, Andreas 1993: Gleichstellungsgesetz für Behinderte, Betrifft JUSTIZ Nr. 34, 87-89.

Jürgens, Gunther 1993: Direkte Demokratie in den Bundesländern, Stuttgart u.a. 1993.

Kammradt, Steffen 1992: Die Verfassungsdiskussion, Frankfurt u.a. 1992.

Kirchhof, Paul 1992: Brauchen wir ein erneuertes Grundgesetz?, Heidelberg 1992.

Kirchhof, Paul 1994: Von der einigenden zur Einheitsverfassung, Universitas 1994, 23-36.

Kloepfer, Michael 1994: Zukunftsbewältigung aus Vergangenheitserfahrung. Die Verfassungskommission wird dem vereinten Deutschland nicht gerecht, FAZ 1.2.1994, 8.

Kokott, Juliane 1993: Der Parteienkompromiß zum Asylrecht vom 15. Januar 1993 - verfassungsrechtliche und völkerrechtliche Anmerkungen, Staatswissenschaft und Staatspraxis, 1993, 197-224.

Kriele, Martin, 1993. Über jeden Grundgesetzartikel einzeln abstimmen, FAZ 21.12.1993, 7.

Kuratorium 1990: Kuratorium für einen demokratisch verfaßten Bund deutscher Länder (Hrsg.), In freier Selbstbestimmung. Für eine Gesamtdeutsche Verfassung mit Volksentscheid, Berlin/Köln/Leipzig 1990.

Kuratorium 1991: Vom Grundgesetz, zur deutschen Verfassung. Denkschrift und Verfassungsentwurf, Baden-Baden 1991.

Landtag NRW 1990: Kommission »Erhaltung und Fortentwicklung der bundesstaatlichen Orndung innerhalb der Bundesrepublik Deutschland - auch in einem Vereinten Europa«, eingesetzt vom Landtag des Landes Nordrhein-Westfalen, Teil 1 (März 1990), Teil 2 (November 1990).

Lieberam, E./Heuer, Uwe-Jens 1993: Die Arbeit der Gemeinsamen Verfassungskommission: Gute Debatten, schlechte Ergebnisse, $D u R$ 1993, 118-133.

Limbach, Jutta/Eckertz-Höfer, Marion (Hg.) 1993: Frauenrechte im Grundgesetz des geeinten Deutschland, Baden-Baden 1993.

Luthardt, Wolfgang 1993: Instrumente direkter Demokratie - Bereicherung der Interessenvermittlung?, in: Hans-Dieter Klingmann/ Wolfgang Luthardt (Hg.) Wohlfahrtsstaat, Sozialstruktur und Verfassungsanalyse, Opladen 1993, 168-188.

Mayer, Thomas 1993: Direkte Demokratie als Chance für die Demokratie, ZRP 1993, 330334.

Merten, Detlef 1992: Verfassungspatriotismus und Verfassungsschwärmerei. Betrachtungen eines Politischen, VerwArch 83 (1992), 283-298.

MöckIi, Silvano 1991: Direkte Demokratie im Vergleich, APuZ B 23/91, 31-43. 
Müller-Bromley, Nicolai 1990: Staatszielbestimmung Umweltschutz im Grundgesetz? Rechtsfragen der Staatszielbestimmung als Regelungsform der Staatsaufgabe Umweltschutz, Berlin 1990.

Narr, Wolf-Dieter, 1991: Es fehlt die Leidenschaft. Der Verfassungsentwurf des Kuratoriums »Bund deutscher Länder«, Blätter für deutsche und internationale Politik 1991, 874-882.

Preuß, Ulrich K. 1990: Der Entwurf der Arbeitsgruppe »Neue Verfassung der DDR Runden Tisches $K J$ 1990,222.

Preuß, Ulrich K. 1991: Die Chance der Verfassunggebung, APuZ B 49/91, 12-19.

Preuß, Ulrich K. 1993a: Plebiszite als Formen der Bürgerbeteiligung, ZRP 1993, 131-138.

Preuß, Ulrich K. 1993b: Auslandseinsätze der Bundeswehr, KJ 1993, 263-277.

Robert, Rüdiger 1993: Umweltschutz, und Grundgesetz, Münster/New York 1993.

Roellecke, Gerd 1991: Brauchen wir ein neues Grundgesetz?, NJW 1991, 2441-2248.

Rubel, Rüdiger 1992, 1993a, 1993b: Das neue Grundgesetz, Teile 1, 2 u. 3, JA 1992, 265-271, 12-18, 296-304.

Rüttgers, Jürgen/Oswald, Eduard (Hg.) 1992: Die Zukunft des Grundgesetzes, Bonn (Schriftenreihe der CDU/ CSU-Bundestagsfraktion Bd. 1), August 1992.

Säcker, Franz Jürger 1994: Das neue Entgeltfortzahlungsgesetz und die individuelle und kollektive Vertragsfreiheit, Arbeit und Recht 1994, 1-12.

Schneider, Hans-Peter 1991: Die Zukunft des Grundgesetzes, in: Rudolf Wildemann (Hg.), Nation und Demokratie, Baden-Baden 1991, 51-70.

Schneider, Hans-Peter 1994: Das Grundgesetz - auf Grund gesetzt. Die Deutschen haben kein Talent zur Verfassungsreform, Das Parlament Nr. 2/1994 (14.1.1994), 8.

Scholz, Rupert 1992: Grundgesetz und Europäische Einigung, NJW 1992, 2593-2601.

Scholz, Rupert 1993a: Aufgaben und Grenzen einer Reform des Grundgesetzes, in: FS Lerche 1993, 65-81.

Scholz, Rupert 1993b: Die Gemeinsame Verfassungskommission, APuZ B 52-53/93, 3-5.

Scholz, Rupert, 1993c: Grundgesetz zwischen Reform und Bewahrung, Berlin/New York.

Seifert, Jürgen, 1990: Klassenkampf von rechts oder Modernisierung des Grundgesetzes, GMH 1990, 614-622.

Seifert, Jürgen 1991: Verfahrensregeln für Streitkultur. Ein Plädoyer für eine Verfassungsdebatte, $K J 1991,362-369$.

Seifert, Jürgen 1992a: Bürgerrechtliche Forderungen an Europa, Vorgänge 120 (6/1992), 97ff.

Seifert, Jürgen 1992b: Vom Lauschangriff zum »Großen Lauschangriff«, $K J$ 1992, 355-363.

Seifert, Jürgen 1993: Die gescheiterte Erneuerung des Grundgesetzes, Vorgänge 123 (3/1993), 90-96.

Storost, Ulrich 1991: Legitimität durch Erfolg? Gedanken zur Dauerhaftigkeit einer Verfassung, Der Staat 30 (1991), 537-547.

Strübel, Michael 1987: Mehr direkte Demokratie, APuZ B 42/87, 17-30.

Thaysen, Uwe 1990: Der Runde Tisch oder: wo blieb das Volk?, Opladen 1990.

Tomuschat, Christian 1990: Wege zur deutschen Einheit. 3. Bericht zur Sondertagung der Vereinigung der Deutschen Staatsrechtslehrer »Deutschlands aktuelle Verfassungslage «, VVDStRL 49 (1990), 70-100.

Wahl, Rainer 1978: Empfehlungen zur Verfassungsreform, AöR 103 (1978), 477-521.

Wiederin, Ewald 1992: Die Verfassunggebung im wiedervereinigten Deutschland. Versuch einer dogmatischen Zwischenbilanz zu Art. 146 GG n.F., AöR 1992, 410-448.

Würtenberger, Thomas 1993: Wiedervereinigung und Verfassungskonsens, JZ 1993, 745-750.

ZDFW (Hg.) 1993: Art. 16a und seine Folgen. Dokumentation, Bonn 1993. 